FRANCISCO GÁLVEZ PIZARRO

ESCUELA DE DISEÑO

FACULTAD DE ARQUITECTURA,

DISEÑO Y ESTUDIOS URBANOS

PONTIFICIA UNIVERSIDAD CATÓLICA

SANTIAGO, CHILE

FGALVEZ@PUC.CL

\section{La bóveda tipográfica: una invitación a contemplar la constelación de tipos}

\author{
The Typographic Vault: An Invitation to \\ Contemplate the Constellation of Types
}

Desde que la producción de fuentes se industrializó, siempre ha habido una obstinación en acoger todos los tipos disponibles en una sola clasificación. Esta iniciativa ha sido abandonada debido a la proliferación de diseños que mezclan estilos a diestra y siniestra. La clasificación más conocida -que hizo Maximilien Vox- posiblemente ha sobrevivido por la adopción de la Association Typographique Internationale (ATypl) en 1962 y el British Standard en 1967. A pesar de ello, dicha clasificación no acoge los diseños contemporáneos. No es un tema que atraiga la atención de los diseñadores en general, pero las clasificaciones son necesarias para entender una complejidad, un recurso mnemotécnico para recordar características de forma con nombres. Cuando en la década de los noventa aparecieron las primeras fuentes experimentales que pusieron en jaque las categorías existentes (aunque hubo algunas que lo hicieron antes, pero no masivamente), se reaccionó con la creación de grupos nuevos, aunque no de un modo muy afortunado. Un ejemplo es el de Lewis Blackwel que, en su libro Tipografía del siglo xx. Remix, de 1998, introduce dos grupos para acoger fuentes que son inclasificables en el modelo de Vox: uno es Contemporary (Contemporáneo) y el otro BC. Beyond Classification (Más allá de la clasificación). Aunque se agradece el intento no es muy útil lo que se puede analizar al respecto. También se ha usado, como un modo de categorizar grupos, conceptos que remiten a la connotación que transmite la forma estilística de una letra, pero esto siempre ha sido un problema ya que las formas no representan siempre una misma idea; son interpretables, reinterpretables o malinterpretables, en definitiva, arbitrarias. Ejemplo de ello son las colecciones que se obtienen por palabras claves en búsquedas de internet como: groovy, terror, modernas, techno, sicodélicas, retro, grunge y quién sabe qué otro término.

No se trata de un problema actual, en las primeras clasificaciones, como la de Francis Thibaudeau (1921-1924), se empleó Antique («antigua» en francés) para referirse a las formas sin serifas o lineales, lo cual ha engendrado nombres que actualmente no hacen sentido, como Antique Olive (1962) de Roger Excoffon o en años anteriores para referirse a serifas cuadrangulares en la Antique (1815) de la fundición Figgins ¿Se imagina usted que en la entomología se usara como criterio el grado de susto que produzcan los insectos en las personas? Bueno, algo parecido pasa cuando un conjunto de diseños se etiqueta con ideas. Eso es para comunicar no para clasificar, además se generan estereotipos que limitan los recursos gráficos para el diseño del mensaje.

Una idea que parece razonable es que la clasificación se concentre en precisar la forma para retenerla, lo cual es una trampa porque el lenguaje tiene problemas para describir fielmente las particularidades de las letras. La tarea de planificar un significado para que sea interpretado por la mayoría de las personas es un ejercicio de la comunicación visual. Tema aparte que no se puede abordar en un par de líneas, aunque existen muchos ejemplos de cómo una misma forma de letra puede representar distintas ideas. Como el caso de
RChD: creación y pensamiento

Universidad de Chile

2019, 4(6)

http://rchd.uchile.cl 
Figura 1. Izquierda: dos fuentes con el nombre

Antique que crean malentendidos en su

connotación: Antique Olive de Roger Excoffon y Antique de Vincent Figgins.

Derecha: distintas aplicaciones que tiene la letra gótica, evidenciando que no solo se relaciona con la Edad Media o Alemania.

De arriba a abajo: Fakir de Underware, Burgundica de Gerrit Noordzij y Vega Fraktur de Rodrigo Valenzuela.

1. Gerrit Noordzij es un diseñador holandés que escribió un texto en 1982 llamado The stroke of the pen: fundamental aspects of western writing en el que explica la naturaleza constructiva del trazo.

\section{Antique Olive Death Metal}

\section{ANTIQUE Síos es mi copiloto}

\section{Bajada por atrás}

la letra gótica. Todos los occidentales tenemos una idea más o menos cliché de lo que es la letra gótica: principalmente, que se asocia a la Edad Media o que es un tipo de letra que proviene de Alemania, pero cuando se revisa su historia descubrimos que se usó en varios lugares de Europa, sobre todo en Francia. A pesar de que apareció en el siglo XII, hoy tiene decenas de aplicaciones. Basta verla en grupos de Death Metal, en rótulos de camiones y buses rurales de Sudamérica o en las barras de fútbol. Así la forma de las letras góticas no representa intrínsecamente la Edad Media ni Alemania, ya que ha sido resignificada en distintos ámbitos.

Por eso es difícil clasificar mediante el significado.

\section{El cubo de Noordzij}

Esta propuesta no considera nada nuevo, no es clasificar en estricto rigor sino una forma de recorrer o navegar la complejidad de las formas estilísticas de las letras. Tal vez de un modo más sencillo y se basa, en parte, de la teoría desarrollada por Gerrit Noordzij ${ }^{1}$ sobre el concepto de contraste (o diferencia entre las partes finas y gruesas de una misma letra) generado por la manipulación de plumas y pinceles. En su libro El trazo. Teoría de la escritura, Noordzij plantea - con su vehemente y provocador punto de vista- una interesante teoría sobre la forma de las letras de acuerdo a dos tipos principales de contrastes: uno generado por la pluma de punta biselada, denominado contraste por traslación y otro que se obtiene con la presión de la pluma de punta flexible, Ilamado contraste por expansión. Un tercer tipo, el contraste por rotación, conviviría con los dos tipos anteriores, pero no complejicemos las cosas, quedémonos con esas dos ideas.

Si bien la intención de configuración de tipos que se expondrá aquí no reemplaza dicha teoría, sí pretende hacer nuevas preguntas. En este sentido, la aportación de lo expuesto en el libro de Noordzij es fundamental para esta propuesta y dista de ser completa.

Además, no podemos olvidar la idea que planteó Alan Turing en 1952, respecto a que todo modelo es una simplificación y una idealización y que por ello siempre será una falsificación. A pesar de ello los modelos siguen siendo útiles cuando se interiorizan.

\section{Cómo entender miles de estilos}

Primero, hay que tener claro algunos conceptos que nos permitan navegar en la teoría de Noordzij que es parte importante de la ordenación que se propone acá y la cual he bautizado como «Bóveda tipográfica». Se trata de tres fenómenos formales en los grosores de las letras (ejes $\mathrm{Y}$ y $\mathrm{Z}$ ) con sus respectivas progresiones en varios pasos o interpolación. Existe también un eje $X$, que contiene un incremento del trazo hacia un grosor más pronunciado. 


\section{aаaаaаaаa}

Rango de pesos considerado por Noordzij
Figura 2. Esquema que muestra el rango de pesos considerado por Gerrit Noordzij

Sin embargo, surgen algunas preguntas al momento de querer aplicarlo, como ¿por qué este modelo de interpolación entre contrastes de letras está representado en un cubo? ¿Qué sucedería si la letra «e» se reemplaza por otra, como una «o»? ¿resistiría esa figura como una manera de representación? El modelo original del cubo no considera los pesos livianos o Light; solo va desde un peso regular a otro en negrita. Bajo la perspectiva de la producción actual en el mercado, se echa de menos esa parte de la progresión.

Esta omisión se debe a las proporciones caligráficas de las escrituras tradicionales que analiza Noordzij, ya que nunca se hicieron con trazos finos sino, más bien, regulares y oscuros (humanísticas/cancillerescas y góticas, respectivamente). Ambos pesos se han usado por siglos para textos largos y algunas personas, en la actualidad, toman la mala decisión de usar letras finas para aquello. Algo bastante incómodo de leer.

Ya que el peso remite a un mismo diseño estilístico, no es útil considerarlo en una ordenación de estilos de formas. Cada estilo de letra puede tener con más o menos problemas de diseño una versión negrita o fina. La misma ineficacia sucede con la condensación y expansión de las proporciones. De este modo, el plano del eje X se eliminaría en la «Bóveda tipográfica».

En la parte inferior del cubo de Noordzij se puede notar que las letras «e» con poco contraste (regulares o negritas) tienen la única sutileza de la diferencia de ángulos en que se cortan sus partes inferiores. Situación que no es tan evidente a simple vista y que, además, excluye varias terminaciones que se producen en letras como a, c, f, r por arriba y j, e, y por abajo, en determinados estilos. Así, sería mejor eliminar esa dualidad y dejarla solo como un polo, el del grosor uniforme o monolineal.

Estas observaciones llevarían a plantear otra manera de explorar y simplificar la representación de los contrastes usando un prisma de base triangular en lugar de hacerlo con un cubo.

En el eje Z, por otra parte, se puede apreciar que es muy sutil la interpolación de dicho eje ¿qué sentido tendría hacer una interpolación así? solo explorativo para crear nuevos diseños. Esa transición sería un híbrido entre una pluma biselada y otra pluma de punta flexible o un pincel, es decir, de un contraste por traslación a uno por expansión. Lo que Noordzij denomina pasar, históricamente, de lo clásico a lo clasicista (seguramente se refiere a pasar de la pluma biselada a la pluma de punta flexible) o, desde la antropología, pasar de lo occidental a lo oriental (de la pluma biselada al pincel), relación que es difícil de sostener debido a que en Oriente también se usa la pluma biselada en la escritura del Devanagari. A pesar de ello, no deja de tener un simbolismo interesante. 
Figura 3. Adaptación simplificada del cubo de Noordzij. El defecto que tiene la representación como un hexágono (izquierda), no permite ver el vértice del fondo que es cubierto por el vértice de encima, en el centro. Algunas personas han creado animaciones donde ese punto puede observarse. En él se observa que las letras «e» monolineales tienen una pequeña diferencia en el remate inferior, que se puede interpretar como un detalle del contraste por rotación.

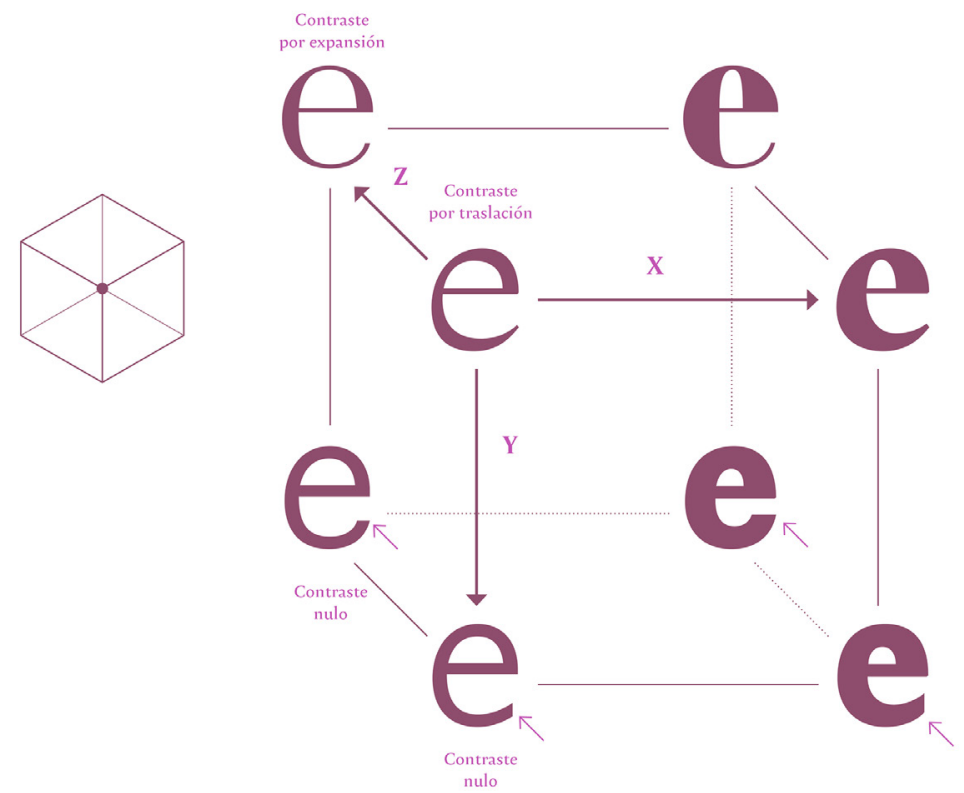

Figura 4. Si el esquema usara la letra «0», podría prescindirse de un vértice para configurar un prisma triangular, más sencillo de comprender, ya que la rotación está implícita en todos los tipos de contrastes.
Contraste por expansión

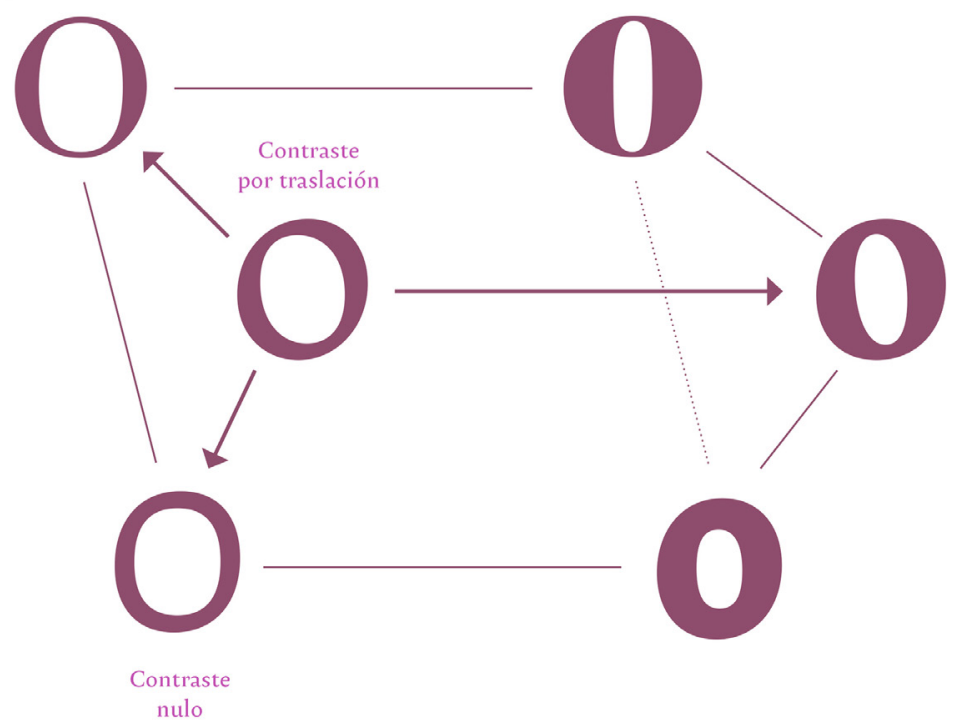

\section{Depurando el modelo de una «bóveda»}

Para realizar la «Bóveda tipográfica» tuve que revisar y ordenar estas ideas y otras que eran difíciles de conciliar en un solo modelo. A pesar de que los contrastes por traslación y por expansión tienen un origen caligráfico, ambos pueden ser susceptibles de tener alteraciones arbitrarias o de tener una aplicación sistemáticamente rígida, por lo cual no se pueden considerar como modelos perfectos (tal vez aquí ayudaría el contraste por rotación, pero eso es 
particularmente de la escritura, no de la tipografía). Así, es mucho más razonable tomar el contraste como una sola categoría - bastante compleja- y el no contraste -mucho menos compleja- como polos de interpolación. La interpolación entre estos extremos puede ser mucho más didáctica para entender la multitud de diseños, aunque por supuesto no todos. A pesar de esto, no se dejan fuera los contrastes por traslación y por expansión en este modelo. Esta idea de complejidad es muy útil sobre todo para la hibridación y mezcla de estilos que tienen los diseños de tipos actuales. Accidentalmente, me encontré con un texto del tipógrafo belga Fernand Baudin, titulado How Typography Works (and why it is important) ${ }^{2}$-irónicamente escrito a mano-, en el cual, en su prólogo, Charles Bigelow menciona sobre las charlas que dio Fernand Baudin en la universidad de Stanford apuntando a temas como «Constellations of Typefaces and Configurations of Texts»3.

Dichos conceptos me hicieron sentido para enriquecer la «Bóveda tipográfica», precisamente por esa analogía que hay con una constelación que implica un conjunto de estrellas - fuentes tipográficas para el caso- que, mediante trazos imaginarios, forman un dibujo que evoca una figura determinada.

En esa línea, la Bóveda tendría esa tarea: la de permitir constelaciones que permitan leer y navegar en dibujos que representen tipos de contrastes, periodos históricos de los estilos, clasificaciones y en general cualquier diseño. Suena ambicioso, pero en realidad, la Bóveda es solo el espacio donde se pueden estudiar esas relaciones y por eso no se trata de una clasificación. Aquí se plantea la construcción de esa esfera que contiene, según mi parecer, las categorías esenciales que permiten hacer la mayoría de los cruces, si no todos. Los polos de la Bóveda serían los siguientes: Serif y Sans, alto contraste y bajo contraste, Estructura variable y Estructura constante. Con estos seis polos o nodos, se podría incluir cualquier forma en el diseño, inclusive de letras Display.

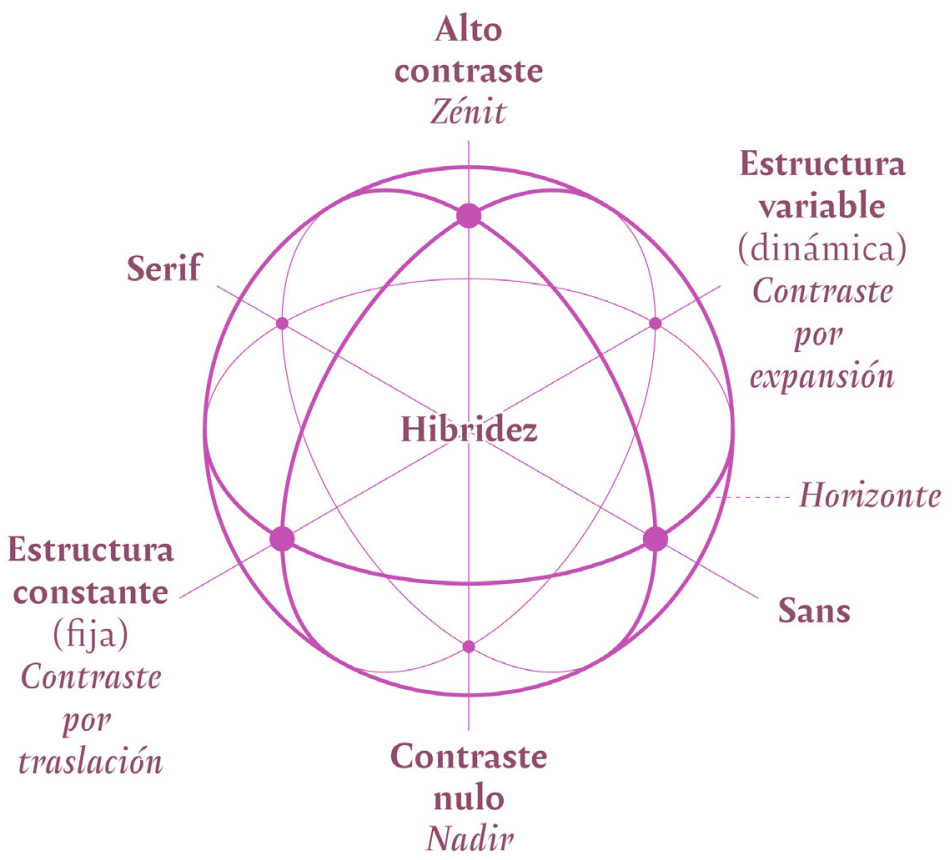

2. Este libro fue escrito originalmente en francés: La Typographie au Tableau Noir (La tipografía en el pizarrón), en 1984.

3. La idea de «constelaciones de caracteres»

$y$ «configuraciones del texto» aparecen en

el artículo 'Constellations \& configurations d'écritures', escrito por Fernand Baudin para una antología sobre tipografía titulada De plomb d'encre, et de lumière publicado en 1982 por la Documentation Française. 
Figura 6. Algunas fuentes que ejemplifican las posiciones en la Bóveda respecto a los polos de alto contraste, el nulo, el Serif y el Sans. Nótese en el caso de Domaine (2) que está más cerca del zénit por tener más contraste que Bodoni Poster (1). En cambio, Rockwell y Neue Hass Unica están más equidistantes del nadir por poseer el mismo contraste nulo (las sutiles diferencias de grosor corresponden a las correcciones ópticas).
Figura 7. Una comparación de letras solo con estructuras opuestas. Al referirse a estructura implica señalar desde el esqueleto hasta las terminaciones (serifas y terminales). Por ejemplo, nótese que Comic Sans (3) es básicamente un esqueleto que no tiene una forma única de trazo, la asta de la «h» no tiene la misma precisión que la asta de la «p», con ligeros cambios, por esta razón no está tan cerca del polo Estructura variable, que sí podrían tener otras fuentes. Sus remates en cambio son todos parecidos, es decir, redondeados. En cambio, Biblon (2) podría estar un poco más cerca del polo porque sus terminales y serifas son más irregulares (observe la parte inferior de la «h»). Por otro lado, Gulliver (1), posee una estructura más estandarizada en sus curvas y serifas que la posiciona más cerca del polo izquierdo, lo mismo que Klavika, pero en el hemisferio de las Sans.
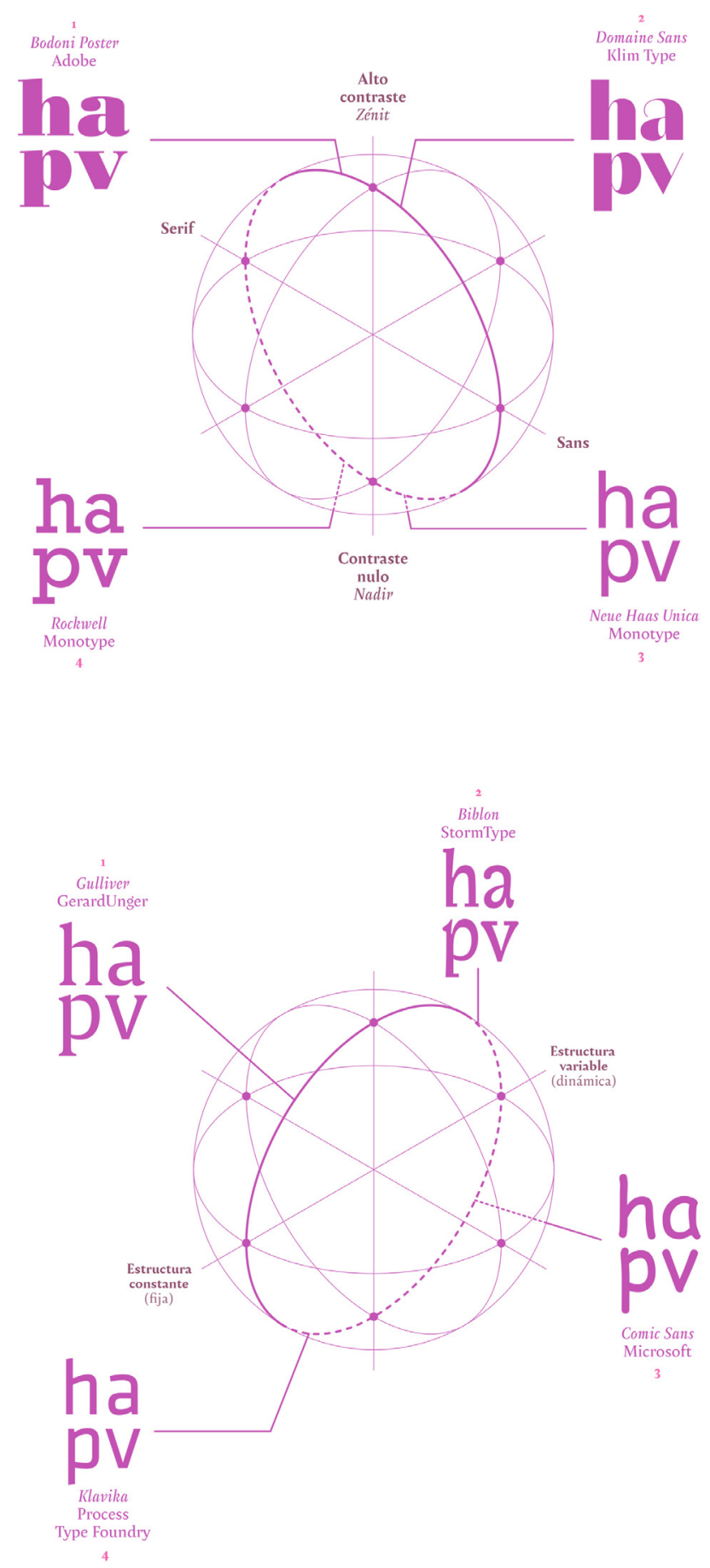


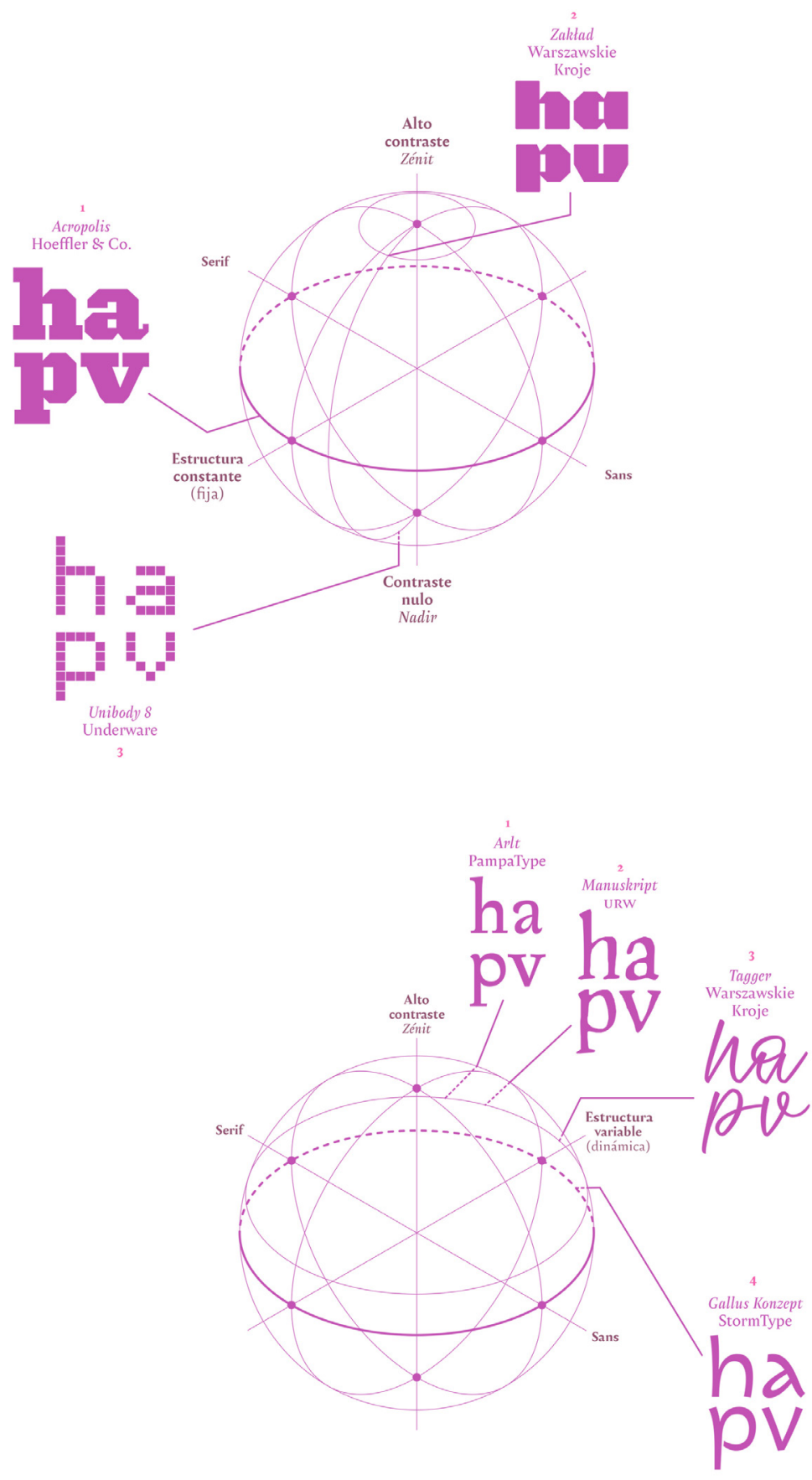

Figura 8. Ejemplos de fuentes cerca del polo Estructura constante o fija, consideran además los polos Serif-Sans y Alto contraste-Nulo, donde se aprecian evidentes formas sistematizadas. No existen curvas y son reemplazadas por diagonales tanto en Acropolis (1) como en Zakład, esta última, más cerca del zénit por poseer un contraste más alto que Acropolis. Unibody 8, puede ser el ejemplo más extremo de una Sans monolineal de Estructura fija, definida por pixeles, es decir, que las fuentes para pantalla estarían muy cerca de este polo; depende de otras características.

Figura 9. Letras de Estructura variable o dinámica, consideran los polos Serif y Sans y el de Alto contraste. La línea secundaria por sobre el horizonte de la Bóveda, permiten ubicar un poco mejor estas posiciones. Tanto para Arlt (1), Manuskript (2) y Tagger (3), el contraste es bastante similar, por ello están ubicados sobre ese horizonte. En cambio, Gallus Konzept (4), tiene un poco de contraste y se ubica en la mitad de entre los polos Alto contraste y Contraste nulo. Nótese que Tagger (3) y Gallus (4) están más cerca del polo Estructura variable que Arlt y Manuskript, debido a su esqueleto. En el esquema, las líneas pasan por detrás de la Bóveda, pues dicho polo queda tapado por la esfera. 
Figura 10. Representación de fuentes Serif-Sans pasando por el centro de la Bóveda donde se ubica Hibridez, es decir, el punto intermedio entre estos polos y que, por lo tanto, contendría todas las fusiones e interpolaciones posibles. Además, está en diagonal porque transcurre desde el punto alto del polo Serif —donde está el polo Alto contraste- hasta bajar al extremo opuesto en el polo Sans y cerca del polo Contraste nulo. Esta sería una constelación de la interpolación Serif-Sans que parte con Baskerville (1), luego las serifas desaparecerán en Chercán (2) hasta que dichas serifas se transforman en el trazo principal de la letra en Infini (3) para luego desaparecer sutilmente en Versa Sans (4) y dejar de existir en Renner ArchiType (5). Optima de Hermann Zapf podría ubicarse en este centro.

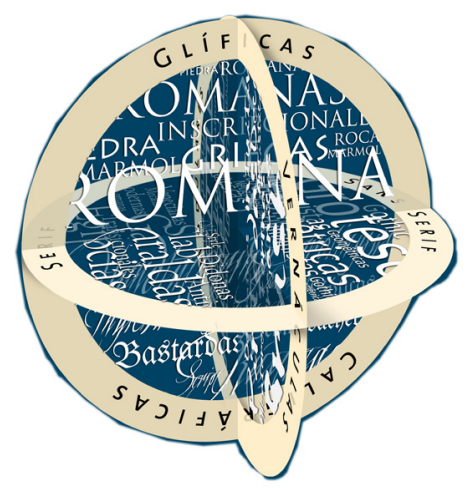

Figura 11. Primera graficación de la idea de una Bóveda tipográfica, ilustración realizada por el autor, Francisco Gálvez, en 1999, usando los software Adobe Dimension y Photoshop.

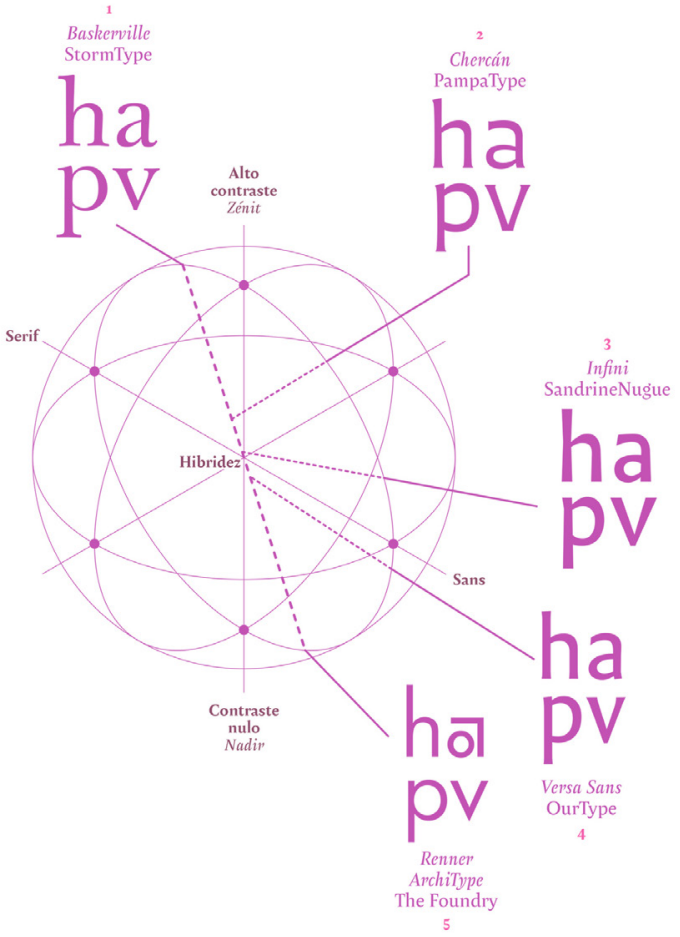

\section{Una invitación a completar}

Para un aprendiz de tipografía o para alguien que comienza a diseñar tipos, esta Bóveda podría ser un punto de partida para luego entender las clasificaciones históricas, las cuales poseen grupos cuyos nombres no representan necesariamente las características formales de los estilos que distinguen. Sin embargo, la Bóveda no reemplaza las clasificaciones, solo intenta acogerlas. Por otro lado, provee un mapa para explorar diseños en partes de los hemisferios que tienen pocos ejemplos.

La Bóveda no debe entenderse como una solución final de todas las formas sino, más bien, un espacio para completar.

Son tantas fuentes las que existen que es imposible incluirlas todas de una vez y actualizarlas constantemente. En este sentido, alguien que sepa de programación podría automatizar una tarea de tal envergadura. César Puertas, diseñador colombiano de tipos, ya hizo un intento de parametrizar las fuentes con los datos y las medidas obtenidas de la información que posee cada archivo tipográfico. Mucho antes Benjamin Bauermeister hizo una catalogación con el sistema PANOSE en 1985. Sin embargo, algunos polos son difíciles de definir con esos datos, como lo son el de Estructura constante y el de Estructura variable.

Con todo, este trabajo, que tuvo su origen a fines de los años noventa, ha tardado bastante en madurar para dar a luz. La incertidumbre y variedad de diseños actuales siempre ha sido la piedra de tope.

Falta mucho por recorrer y discutir. 